\title{
Physics case of the very high energy electron-proton collider, VHEeP
}

\author{
A. Caldwell \\ Max Planck Institute for Physics, Munich \\ E-mail: caldwel l@mpp.mpg.de \\ F. Keeble \\ University College London \\ E-mail: fearghus.keeble.11@ucl.ac.uk \\ E. Simpson Dore \\ University College London \\ E-mail: emma.dore.13@ucl.ac.uk

\section{Wing*i} \\ University College London \\ E-mail: m.wing@ucl.ac.uk
}

\begin{abstract}
The possibility of a very high energy electron-proton (VHEeP) collider with a centre-of-mass energy of $9 \mathrm{TeV}$ has been presented at previous workshops. These proceedings briefly summarise the VHEeP concept, which was recently published, and developments since then, as well as future directions. At the VHEeP collider, with a centre-of-mass energy 30 times greater than HERA, parton momentum fractions, $x$, down to about $10^{-8}$ are accessible for photon virtualities, $Q^{2}$, of 1 $\mathrm{GeV}^{2}$. This extension in the kinematic range to low $x$ complements proposals for other electronproton or electron-ion colliders.
\end{abstract}

XXV International Workshop on Deep-Inelastic Scattering and Related Subjects

3-7 April 2017

University of Birmingham, $U K$

\footnotetext{
* Speaker.

${ }^{\dagger}$ Also at DESY.
} 


\section{Introduction}

Simulations of proton-driven plasma wakefield acceleration [1] have shown that acceleration of electrons to TeV scales over km scales is possible. The AWAKE experiment [2] at CERN is investigating and measuring the effect for the first time. Assuming the current and future investigations at AWAKE are successful, possible applications of the AWAKE acceleration scheme are being considered in which an electron beam is accelerated to high energies over short distances. Making strong use of CERN infrastructure, a very high energy electron-proton (VHEeP) collider [3] has been proposed with an electron energy of $E_{e}=3 \mathrm{TeV}$ and $E_{p}=7 \mathrm{TeV}$, leading to a centre-of-mass energy of about $9 \mathrm{TeV}$.

The proposed scheme for VHEeP leads to a baseline centre-of-mass energy a factor of 30 times higher than HERA and a corresponding extension to low Bjorken $x$ of a factor of 1000 , with a similar extension to high photon virtuality, $Q^{2}$, depending on the luminosity. The layout for VHEeP leads to modest luminosities, with an integrated luminosity during the collider's lifetime of $10-100 \mathrm{pb}^{-1}$. Studies showed that the nature of the strong force and its explanation within QCD is not well understood at values of Bjorken $x$ down to $10^{-8}$, corresponding to $Q^{2}$ of about $1 \mathrm{GeV}^{2}$. It is expected that effects of saturation of the structure of the proton and hadronic cross sections will be observed given the unphysical nature of various models when extrapolated to VHEeP energies. This can be studied in inclusive deep inelastic scattering events, by measuring the total photonproton cross section, as well as tagging vector mesons. Given the rise of the cross sections to low Bjorken $x$, even with modest luminosities, numerous events are expected in all of these reactions. Exotic, high- $Q^{2}$ physics can also be studied at VHEeP, with particular sensitivity to leptoquark production up to the kinematic limit of $9 \mathrm{TeV}$, as well as quark substructure.

The physics case of VHEeP developed in the original publication [3] was briefly described in the previous paragraph, with the rest of the proceedings concentrating on developments since. In Section 2, recent developments are discussed, in particular the use of modern Monte Carlo tools and comparison with the previous studies. In Section 3, a workshop dedicated to the particle physics case for VHEeP is briefly summarised. The proceedings are concluded in Section 4.

\section{Recent studies and Monte Carlo development}

Studies published previously [3] used the ARIADne [4] Monte Carlo programme to investigate $e p$ collisions at $9 \mathrm{TeV}$. The ARIADNE Monte Carlo programme is written in Fortran and was used standalone and so a more modern framework and programmes were investigated. The ARIADNE predictions were also made using the CTEQ2L [5] proton parton density functions (PDFs); more modern Monte Carlo programmes are interfaced to LHAPDF [6] and so allow the use of more recent PDFs. The RIVET toolkit (Robust Independent Validation of Experiment and Theory) [7] is a system for validation of Monte Carlo generators and so once a routine has been developed to investigate VHEeP collisions, then several Monte Carlo programmes can be used; the Monte Carlo programmes can also be compared to existing data and so their model(s) validated.

Within the Rivet framework, simulations of high energy ep collisions were performed with the RAPGAP [8] and HeRWIG [9] Monte Carlo programmes. These were run with more modern proton PDFs than used for ARIADNE, namely CT10 [11], MRST2004 FF4 LO [12] and NNPDF 
3.0 LO [10]. The programmes could also simulate events at very low Bjorken $x$, whereas the results from previous simulations with ARIADNE were restricted to a minimum Bjorken $x$ of $10^{-7}$. The results of the simulations and comparison to previous results are shown in Fig. 1. The variables photon virtuality, $Q^{2}$, and inelasticity, $y$, are shown for ARIADNE (Fig. 1(a,c)) and RAPGAP 3.3 and HeRwig 7.0 (Fig. 1(b,d)). The shapes of the three simulations are similar versus $Q^{2}$, most clearly shown for RAPGAP 3.3 and HERWIG 7.0. The shapes are also similar versus $y$, although a difference between RAPGAP 3.3 and HERWIG 7.0 can be seen.
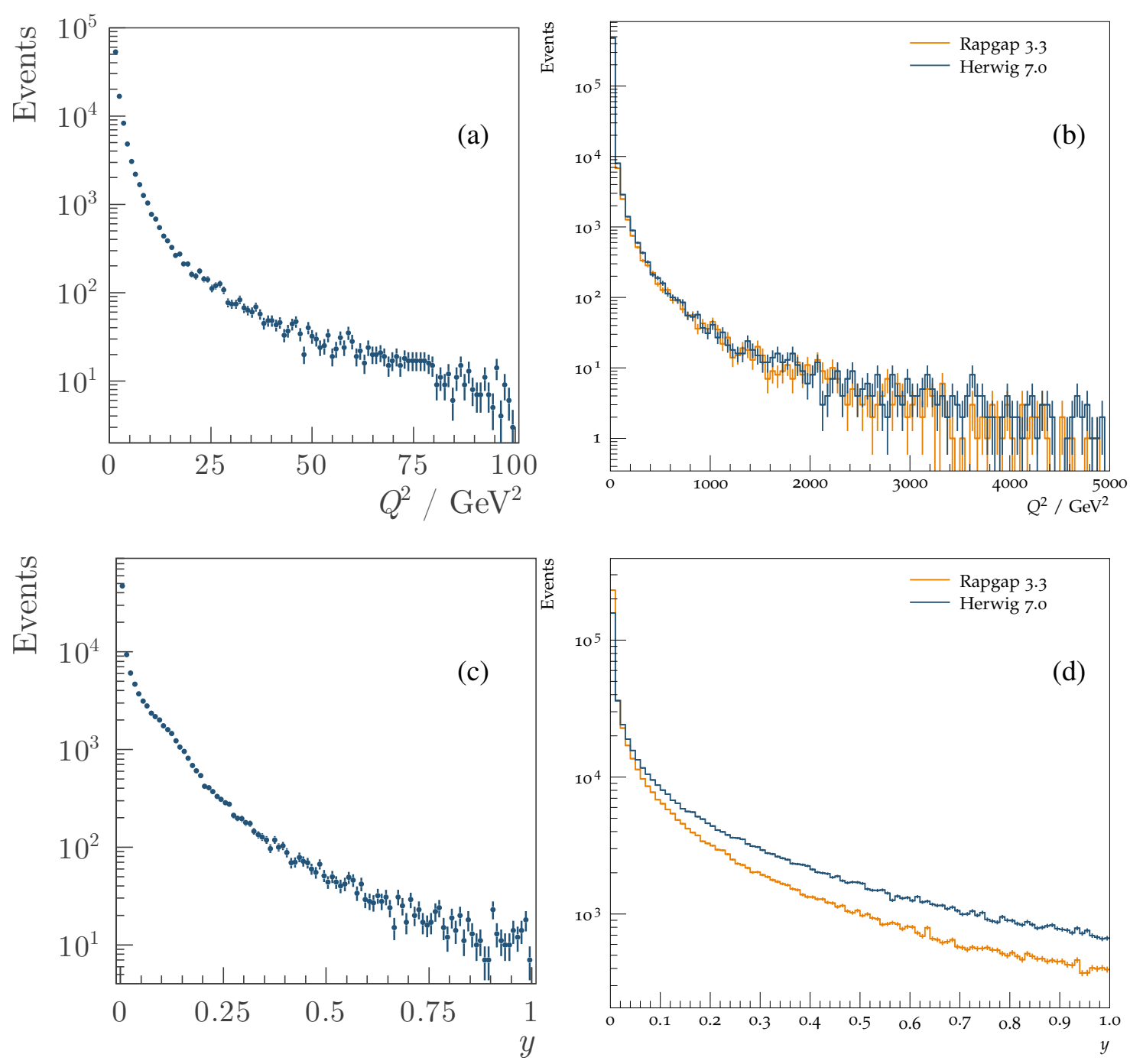

Figure 1: Kinematic distributions for (a,b) photon virtuality, $Q^{2}$, and (c,d) inelasticity, $y$, for a small sample of events at VHEeP. (a,c) show 100k events generated [3] using the ARIADNE Monte Carlo programme with the CTEQ2L proton PDFs for $Q^{2}>1 \mathrm{GeV}^{2}, x>10^{-7}$ and $W^{2}>5 \mathrm{GeV}^{2}$. (b,d) show 500k events generated using either RAPGAP 3.3 (orange line) or HERWIG 7.0 (blue line) Monte Carlo programmes with the NNPDF 3.0 LO proton PDFs for $Q^{2}>1 \mathrm{GeV}^{2}$.

A further comparison between RAPGAP 3.3 and HERWIG 7.0 and between the central values 
of different proton PDFs, CT10 LO, MRST2004 FF4 LO and NNPDF3.0 LO, using RAPGAP 3.3 is shown for the cross section versus Bjorken $x$ in Fig. 2. The predictions from RAPGAP 3.3 with different proton PDFs are very similar for Bjorken $x>10^{-5}$, where they are well constrained by current data. The PDFs deviate from each other to lower Bjorken $x$, where they are extrapolated under different assumptions. Given the lack of data at very low Bjorken $x$, the true uncertainty is larger than the spread in these predictions, however, these predictions can be used as representative event simulations. Differences between HERWIG 7.0 and RAPGAP 3.3, using the same proton PDF, are observed over the full Bjorken $x$ range, even in the region well constrained by data. This indicates differences in the simulations which should be investigated further. It should be noted that the Monte Carlo simulations can be validated with HERA data within RIVET, although the number of analyses from H1 and ZEUS number just a few at the time of writing. It is hoped this will improve in the future and such differences between simulations can be understood.

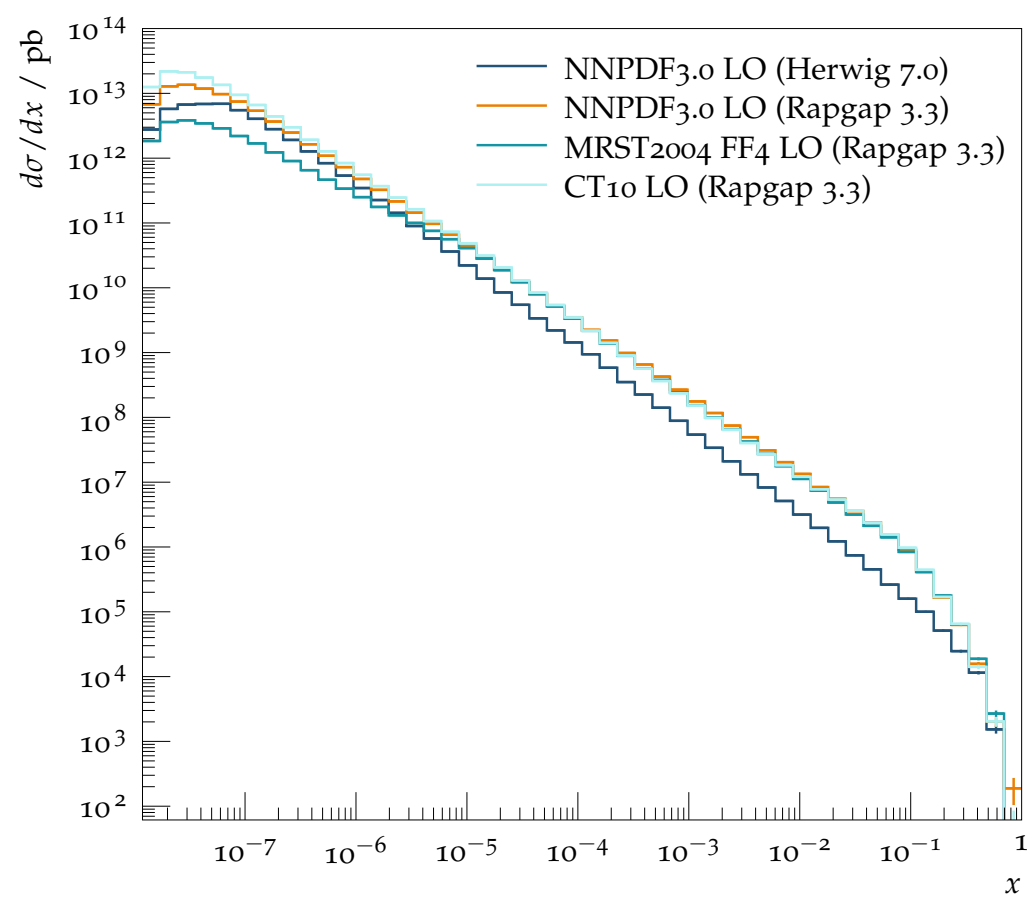

Figure 2: Comparison of the Bjorken $x$ distribution when using either RAPGAP 3.3 (orange line) or HERWIG 7.0 (dark blue line) Monte Carlo programmes with the NNPDF 3.0 LO proton PDFs and also the RAPGAP 3.3 Monte Carlo programme with either MRST2004 FF4 LO or CT10 LO proton PDFs.

\section{VHEeP workshop}

A workshop [13], "Prospects for a very high energy ep and eA collider", was held soon after the DIS 2017 workshop. The workshop covered two full days, held in the Max Planck Institute for Physics, Munich, with experts in the field invited to further explore the particle physics case for VHEeP. The VHEeP baseline parameters with ranges which physics studies should consider are the following: 
- collisions are nominally electron-proton, but electron-ion (e.g. electron-lead) collisions are foreseen;

- there should be the possibility of accelerating positrons and having positron-proton collisions;

- polarisation of the lepton beam should be possible and polarised protons should be considered;

- the nominal energies are $E_{e}=3 \mathrm{TeV}$ and $E_{p}=7 \mathrm{TeV}$, giving a centre-of-mass energy of $\sqrt{s}=9.2 \mathrm{TeV}$

- the electron beam energy can be varied in the range 0.1 to $5 \mathrm{TeV}$, giving $\sqrt{s}=1.7-11.8 \mathrm{TeV}$;

- the integrated luminosity is in the range $10-1000 \mathrm{pb}^{-1}$.

If different parameters are required, this will need to be considered with the constraints imposed by the acceleration scheme.

A brief summary of the workshop is given here and the interested reader is referred to the slides on the workshop web-page [13]. The workshop started with an introduction to plasma wakefield acceleration and its application to higher energy physics (Caldwell), a report on the status of AWAKE (Muggli) and an introduction to VHEeP (Wing). Of particular note were the presentations on QCD and hadronic cross sections (Bartels, Mueller, Schildknecht and Stodolsky) in which theoretical expectations show that saturation will be observed at VHEeP and will also be at a scale where QCD calculations are perturbative. Low- $x$ physics was related to other areas, including cosmic rays (Stasto) and black holes and gravity (Erdmenger), as well as being used as a testing-bed for new physics descriptions (Dvali and Kowalski). The needs of polarisation and electron-ion physics were discussed (Aschenauer and Mäntysaari) as well as what has been learnt from the HERA data at low $x$ (Myronenko). Finally, the status of Monte Carlo simulations for $e p$ and $e A$ physics was presented (Plätzer) as well as the general kinematics and challenges faced by the detector as well as the use of simulations (Keeble).

\section{Summary}

These proceedings have highlighted recent progress on studies for a very high energy electronproton (VHEeP) collider since the proposal was published. Simulations have been extended and can be used to investigate the physics potential and aid design of the detector and accelerator. A workshop to further investigate the physics potential of electron-proton and electron-ion collisions at very high energies was held in June and was here briefly summarised. It should also be investigated how VHEeP could fit into the future world particle physics programme and complement other similar projects investigating deep inelastic scattering.

\section{References}

[1] A. Caldwell et al., Nature Phys. 5 (2009) 363;

A. Caldwell and K. Lotov, Phys. Plasmas 18 (2011) 103101. 
[2] AWAKE Coll., R. Assmann et al., Plasma Phys. Control. Fusion 56 (2014) 084013;

AWAKE Coll., A. Caldwell et al., Nucl. Instrum. Meth. A 829 (2016) 3;

AWAKE Coll., E. Gschwendtner et al., Nucl. Instrum. Meth. A 829 (2016) 76;

[3] A. Caldwell and M. Wing, Eur. Phys. J. C 76 (2016) 463.

[4] L. Lönnblad, Comp. Phys. Comm. 71 (1992) 15; L. Lönnblad, Z. Phys. C 65 (1995) 285.

[5] CTEQ Coll., J. Botts et al., Phys. Lett. B 304 (1993) 159.

[6] A. Buckley et al., Eur. Phys. J. C 75 (2015) 132;

D. Bourilkov, R.C. Group and M.R. Whalley, arXiv:hep-ph/0605240; M.R. Whalley, D. Bourilkov and R.C. Group, arXiv:hep-ph/0508110.

[7] A. Buckley et al., Comp. Phys. Comm. 184 (2013) 2803.

[8] RAPGAP hepforge site, https: / / rapgap .hepforge.org; H. Jung, Comp. Phys. Comm. 86 (1995) 147.

[9] J. Bellm et al., Eur. Phys. J. C 76 (2016) 196.

[10] NNPDF Coll., R.D. Ball et al., JHEP 1504 (2015) 040.

[11] H.-L. Lai et al., Phys. Rev. D 82 (2010) 074024.

[12] A.D. Martin et al., Phys. Lett. B 636 (2006) 259.

[13] Workshop on "Prospects for a very high energy ep and $e A$ collider", MPI Munich, June 2017, https://indico.mpp.mpg.de/event/5222/overview 